\title{
A Design of Navigation System Using Stratospheric Airships in South Korea
}

\author{
Eun Sung Lee* \\ Department of Mechanical and Aerospace Engineering, UCLA, USA \\ Sebum Chun**, Young Jae Lee*** \\ Department of Aerospace Engineering, \\ Konkuk University, Seoul 143-701, Korea \\ Jung Hur**** \\ Department of Electronics Engineering, \\ Konkuk University, Seoul 143-701, Korea \\ Tae Sam Kang*** \\ Department of Aerospace Engineering, \\ Konkuk University, Seoul 143-701, Korea
}

\begin{abstract}
For a relatively small country like Korea, a radionavigation system using airships can be considered, which is to provide the navigation service utilizing the stratospheric airships that are deployed in the stratosphere at the altitude of around $20-23 \mathrm{~km}$, and which is an independent or a back-up radionavigation system other than current GPS or GLONASS. In this paper, a feasibility study on the constellation of stratospheric airships for the navigation system has been performed. A measure of a geometrical condition between a receiver and navigation transmitters, called the DOP (Dilution of Precision), determines the resulting positioning error of the navigation system, if the error of range measurement is predictable. Therefore, with assumption that the range measurement error of the stratospheric airship navigation system is quite similar to GPS, the several DOP values have been used to evaluate the performance of the navigation system with comparing with the DOP values of GPS as the reference values. To provide the position information of the navigation transmitters to users, a receiver cluster system fixed on the ground, called an IGPS (inverted GPS), is proposed, and the error is also evaluated using the DOP values. Five areas around five major cities in South Korea have been selected, and then by numerical simulations the DOP values are compared those of GPS to assess the performance of the proposed navigation system using stratospheric airships. The possible frequency bands have been proposed, and then link budget of the navigation transmitter has been analyzed for the proposed navigation system.
\end{abstract}

Key Words : GPS, Radinavigation, DOP, IGPS, Link Budget

\footnotetext{
* Post Doctor

** Research Assistant

*** Professor

E-mail : younglee@konkuk.ac.kr Tel : 02-450-3358 Fax : 02-444-6670

**** Professor
} 


\section{Introduction}

As for typical radionavigation systems, there are GPS (Global Positioning System) that is operated by the USA and GLONASS (Global Navigation Satellite System) by Russia. Nowadays most people are considering first using GPS as a position, velocity, and timing measurement device. There had been various kinds of radionavigation systems before the above-mentioned satellite navigation systems, but the endless possibility in practical applications has resulted in unprecedented popularity and widespread usage of GPS and GLONASS. Of such extensive applications, the precise timing information feature has already been put into regular practice in the main communication network of many countries. Also the application of GPS linked with the design of the precise geographic information system means that GPS now serves not only a simple purpose of survey but has come to have significance as a main infrastructural system of a nation. Therefore major countries such as European countries and Japan have already been performing various projects on satellite navigation systems in two directions. The first one is constructing a GPS augmentation system to use the full performance of GPS. EGNOS (European Geostationary Navigation Overlay System) of Europe and MSAS (MTSAT Satellite Augmentation System) of Japan are the examples. Basically those are the network systems to increase accuracy, integrity, and availability of GPS and GLONASS for various civilian applications. The second one is to have their own navigation systems to replace GPS and GLONASS that are originally developed for military purpose. Galileo by European countries is expected as the first independent civilian satellite navigation system other than GPS and GLONASS.

As for the type of a radionavigation system that can replace GPS in a relatively small country like Korea, roughly three possibilities can be considered: the satellite navigation system using geosynchronous and geostationary orbit satellites, the navigation system using stratospheric airships, and the ground radionavigation system using the wireless communication network that has already been set up for mobile communication on the ground. Of those three possibilities, the navigation system utilizing the stratospheric airships that are deployed in the stratosphere at the altitude of approximately $20-23 \mathrm{~km}$ is considered in this paper. These kinds of stratospheric airships can be maintained at every one to two years and considered to have about 10 year life span. High efficiency solar electric batteries are to be used as the power source and it is expected to be equipped with an unmanned automatic system. The system can be used not only for navigation but also for other applications, i.e., remote sensing observation as well as a base station of wireless communication system.

This paper focuses on an array of navigation airships with the purpose to design the constellation of the navigation system to serve the South Korea region, and on an airship position determination using so-called the IGPS (Inverted GPS) method. Finally, the frequency allocation, the transmitting antenna, and the link budget of the navigation transmitting system have been analyzed.

\section{Navigation System Design Using Stratosheric Airships}

In order to design a navigation system appropriate for the South Korea region using stratospheric airships, we analyze on the position accuracy, which has to be considered prior to a navigation system design, and then we deal with the method to design the navigation system using stratospheric airships and developed a methodology to determine the constellation of the airships.

\section{Position Accuracy}

The most important part in the navigation system is to evaluate the position error that can be predicted with the following Equation (1) ${ }^{1.2}$ 
where

\section{DOP: Dilution of Precision}

In the above Equation (1), the range measurement error is the error according to the condition that the signal leaving the navigation transmitter arrives at the receiver, and DOP is a kind of magnification factor according to the geometrical condition of the transmitters and the receiver, which changes as to their relative position as shown in Figure 1 (a) and (b). In other words, even if the range measurements errors are the same, the final positioning errors could be different with different geometrical conditions. In the figure, the widths of range measurements represent the errors of the range measurements, and the shadowed areas represent the sizes the resulting positioning error.

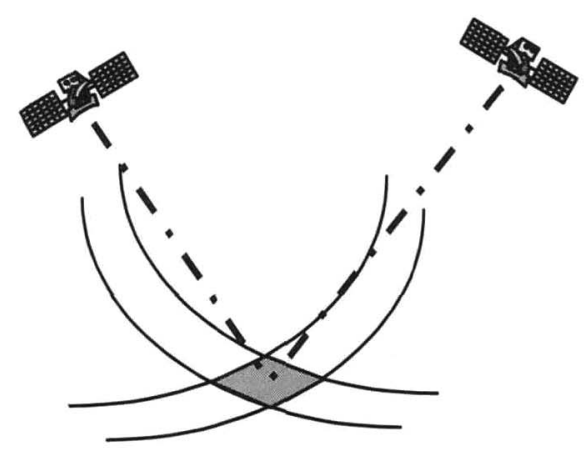

(a) Good Positioning Condition of Satellites and a Receiver (Smaller DOP)

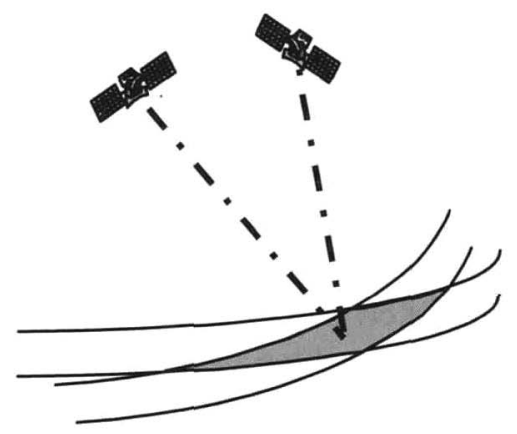

(b) Poor Positioning Condition of Satellites and a Receiver (Higher DOP)

\section{Fig. 1. Basic Concept of DOP and Position Error}

Calculation of the DOP is explained as follows ${ }^{2}$ :

$$
\Delta \rho=H \Delta x
$$

Equation (2) expresses the relationship between the pseudorange offset $\Delta \rho$ and the user coordinate offset $\Delta x$ in terms of matrix $H$. The user coordinate and a receiver clock bias can be obtained by calculating $\Delta x$. W can use the least square method to obtain the following solution.

$$
\Delta x=\left(H^{T} H\right)^{-1} H^{T} \Delta \rho
$$

The pseudorange offset can be expressed as follows.

$$
\Delta \rho=\rho_{T}-\rho_{L}+d \rho
$$

where

$\rho_{T}$ : true range between satellites and a receiver,

$\rho_{L}$ : pseudorange for the linearization reference point,

$\Delta \rho$ : pseudorange offset from the reference point. 
A similar method can be used to describe the user coordinate offset.

$$
\Delta x=x_{T}-x_{L}+d x
$$

where

$x_{T}:$ true receiver coordinates and clock bias,

$x_{L}:$ reference receiver coordinates and clock bias for the linearization with respect to the reference point.

$d x$ : position offest from the reference point

Using the relationship between the Equations (3), (4), and

$$
\left(x_{T}-x_{L}\right)=\left(H^{T} H\right)^{-1} H^{T}\left(\rho_{T}-\rho_{L}\right) \text {, }
$$

we obtain the following equation.

$$
d x=\left[\left(H^{T} H\right)^{-1} H^{T}\right] d \rho=K d \rho
$$

where

$$
K=\left[\left(H^{T} H\right)^{-1} H^{T}\right]
$$

Equation (6) shows that the pseudorange error is reflected on the user coordinate and the receiver clock error.

If we consider the pseudorange error as the random variable, according to the Equation (6), $d x$ is expressed as the random variable related with $d \rho$. Error vector $d \rho$ is generally supposed to consist of the joint Gaussian distribution with zero mean. If geometrically determined, $d x$ is a joint Gaussian with zero mean as well. The covariance of $d x$ can be obtained by averaging the multiplication of $d x$ and $d x^{T}$.

$$
\operatorname{cov}(d x)=E\left(d x d x x^{T}\right)
$$

where $E$ represents an expectation operator, and Equation (7) can be written as follows:

$$
\operatorname{cov}(d x)=\left(H^{T} H\right)^{-1} \sigma_{R}^{2}
$$

where $\sigma_{R}$ represents the range measurement error.

According to Equation (8), we can see that the covariance of the bias error of position and receiver clock bias is affected only by $\left(H^{T} H\right)^{-1}$.

The DOP components of GPS are determined by the ratio of combination of $d x$ covariance components and $\sigma_{R}$. The ratio of the square roots of the sum of all the covariance components and $\sigma_{R}$ is generally called the GDOP (Geometric Dilution of Precision) and defined as below.

$$
G D O P=\frac{\sqrt{\sigma_{x_{u}}^{2}+\sigma_{y_{u}}^{2}+\sigma_{z_{u}}^{2}+\sigma_{c t_{b}}^{2}}}{\sigma_{R}}
$$

where $\sigma_{x_{u}}^{2}, \sigma_{y_{u}}^{2}, \sigma_{z_{u}}^{2}$, and $\sigma_{c_{t}}^{2}$ represent the covariance components of the user position and the receiver clock bias errors respectively.

As seen later, a large value of DOP tends to increase the final position error. Hence, it is to be considered as an amplification factor influenced by the geometrical condition of a user receiver and navigation transmitters as Equation (8). For convenience the several DOPs are defined and summarized as Table 1. 
Table 1. Various DOPs

\begin{tabular}{|c|c|}
\hline DOPS & Description \\
\hline \hline GDOP & Geometric DOP \\
\hline PDOP & Position DOP \\
\hline HDOP & Horizontal DOP \\
\hline VDOP & Vertical DOP \\
\hline TDOP & Time DOP \\
\hline
\end{tabular}

The correlation among various DOP values can be expressed as follows. ${ }^{1.2}$

$$
\begin{aligned}
& \sqrt{\sigma_{x_{n}}^{2}+\sigma_{n}^{2}+\sigma_{n_{n}}^{2}}=P D O P \times \sigma_{R} \\
& \sqrt{\sigma_{x_{n}}^{2}+\sigma_{n,}^{2}}=H D O P \times \sigma_{R} \\
& \sigma_{z_{n}}=V D O P \times \sigma_{R} \\
& \sigma_{u_{n}}=T D O P \quad \times \sigma_{R}
\end{aligned}
$$

\section{Errors in Navigation System Using Stratospheric Airships}

The stratospheric airships used for a navigation system can be operated at the altitude of approximately $20-23 \mathrm{~km}$ where little airflow is present. They receive their position information from the ground stations and send it to user receivers through the navigation message in the transmitting signal. Range measurement errors could also occur when a ground station receiver receives the signal to obtain the positions of the stratospheric airships. This will be a part of error budget of the final position error of the navigation system.

Figure 2 shows the procedure that the airship position is estimated at the ground control station and is transmitted to users via the airships. Furthermore, while GPS satellite the ranging signal passes through the ionosphere, the stratosphere, and the troposphere, the stratospheric airship signal passes through the troposphere and small part of the stratosphere only. The error budgets of the position errors of GPS and the stratospheric airships are summarized as Table $2^{3}$ and Table 3. Most other components except the ionospheric error are almost the same. Since the stratosphere is assumed to be stable, the positioning error of the airship is assumed as much as $3.0 \mathrm{~m}$. Hence, all the error components except the two errors are assumed the same values. As we can see from Table 3 , when the vertical and the horizontal errors are compared with the case of GPS as in Table 2 we have outstanding positioning results from the stratospheric airship system.

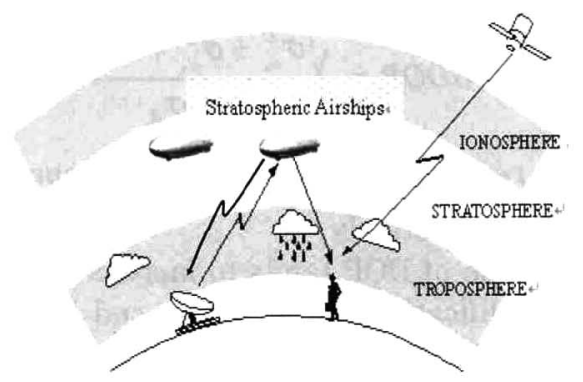

Fig. 2. Signal Flows of the Airship Navigation System 
Table 2. Typical Error Budget of GPS (SA-off, C/A-code)

\begin{tabular}{|c|c|c|c|}
\hline \multirow{2}{*}{ Error Source } & \multicolumn{3}{|c|}{$1 \sigma \mathrm{rms}$ error $(\mathrm{m})$} \\
\cline { 2 - 4 } & Bias & Random & Total \\
\hline \hline Satellite orbit & 2.1 & 0.0 & 2.1 \\
Satellite clock & 2.0 & 0.7 & 2.1 \\
Ionospheric delay & 4.0 & 0.5 & 4.0 \\
Tropospheric delay & 0.5 & 0.5 & 0.7 \\
Multipath & 1.0 & 1.0 & 1.4 \\
Receiver Measurement & 0.5 & 0.2 & 0.5 \\
\hline UERE & 5.1 & 1.4 & 5.3 \\
Filtered UERE & 5.1 & 0.4 & 5.1 \\
\hline Vertical error (VDOP = 2.5) & & & 12.8 \\
Horizontal error (HDOP =2.0) & & & 10.2 \\
\hline
\end{tabular}

Table 3. Error Budget of Stratospheric Airships

\begin{tabular}{|c|c|c|c|}
\hline \multirow{2}{*}{ Error Source } & \multicolumn{3}{|c|}{16 rms error (m) } \\
\cline { 2 - 4 } & Bias & Random & Total \\
\hline \hline Airship position & 3.0 & 0.0 & 3.0 \\
Airship clock & 2.0 & 0.7 & 2.1 \\
Ionospheric delay & 0.0 & 0.0 & 0.0 \\
Tropospheric delay & 0.5 & 0.5 & 0.7 \\
Multipath & 1.0 & 1.0 & 1.4 \\
Receiver Measurement & 0.5 & 0.2 & 0.5 \\
\hline UERE & \multirow{2}{*}{3.8} & 1.3 & 4.0 \\
\hline Vertical error (VDOP = 2.5) & & & 10.0 \\
Horizontal error (HDOP =2.0) & & & 8.0 \\
\hline
\end{tabular}

\section{Navigation System Using Stratospheric Airships}

Two phases are to be considered in constructing the navigation system using stratospheric airships. The first phase is developing a GPS augmentation system. This is a kind of a Differential GPS (DGPS) error correction data transmit system that provides users with the error correction data calculated at the ground reference station, which enables the high accuracy DGPS positioning services. The second phase is to use the airship as an alternative or a back-up navigation system other than GPS. The airship is equipped with a navigation transmitter to provide the radionavigation service. Figure 3 shows the rough sketch of the system.

One of the important factors in the radionavigation system using stratospheric airships is to estimate the airship positions, or the transmitter positions. These estimated airship position data are transmitted to users in the form of navigation message within the ranging signal as the same as GPS. In order to obtain the airship position data the following method shown as Figure 4 could be used. In this method, the position signals of the airships are observed at the fixed observation points on the ground, and are sent to the master control station, where the data are combined to estimate the airship positions. This method is called IGPS (Inverted GPS) ${ }^{4}$ since it is different from ordinary GPS kind of navigation methods. That is, the navigation transmitter signals are received by a receiver whose positions are known and the position of the navigation transmitter is computed through an inverse process. 


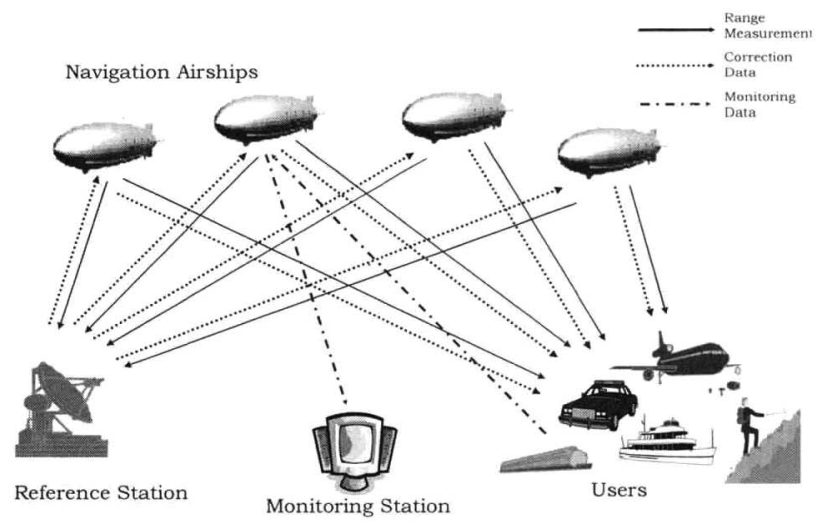

Fig. 3. Navigation System Using Stratospheric Airships

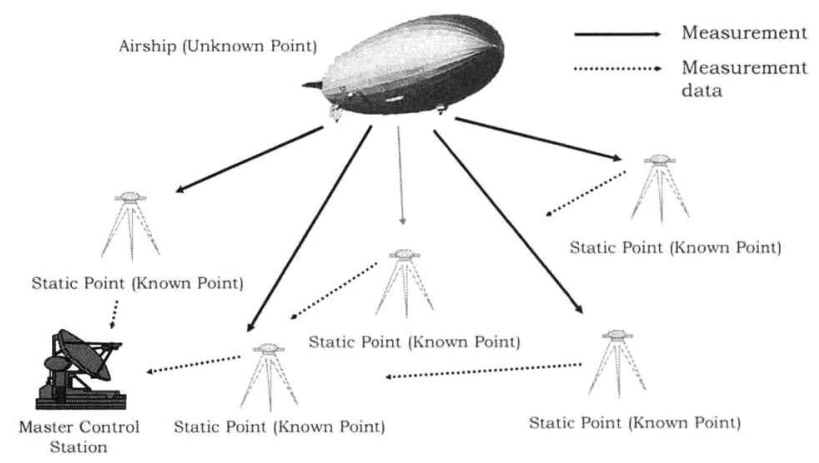

Fig. 4. Concept of the IGPS Method for Estimating Airship Positions

\section{The Simulation of Stratospheric Airships}

Figure 5 shows the visible range of the stratospheric airship at the altitude of $22 \mathrm{~km}$ of Daejun with respect to various mask angles. The radius of the visibility range is about 530 $\mathrm{km}$ when the mask angle is set at 0 degrees, about $210 \mathrm{~km}$ at 5 degrees, about $110 \mathrm{~km}$ at 10 degrees, and about $80 \mathrm{~km}$ at 15 degrees. The mask angle is set to 15 degrees through the simulation.

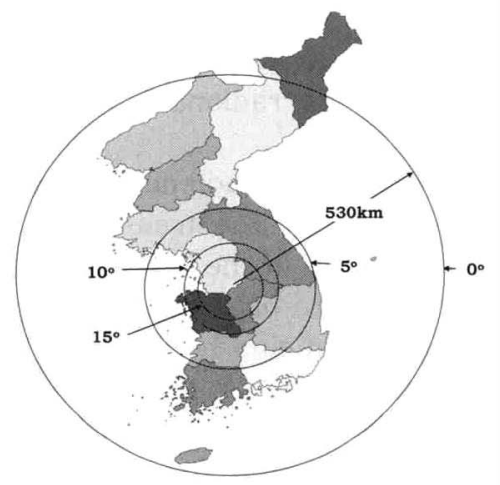

Fig. 5. The Visible Range of the Stratospheric Airship with Varying Mask Angles from Daejeon 


\section{Basic Simulation of The Navigation System Using Stratospheric Airships}

On the basis of the data regarding the visibility range with respect to the mask angle of 15 degrees, two scenarios are simulated: the first scenario is deploying a single ground receiver and multiple airships as Table 4 (Cases 1 and 2), which is a navigation system. The other is deploying a single airship and multiple ground receivers (Cases 3 and 4), which is an airship tracking system for the purpose providing the position information through navigation message of each airship to users to solve their navigation solutions.

Table 4. Four Different Cases of Simulation

\begin{tabular}{|c|c|c|c|}
\hline Categories & Cases & Locations of Receivers & Locations of Airships \\
\hline \hline \multirow{2}{*}{$\begin{array}{c}\text { Navigation } \\
\text { System }\end{array}$} & Case 1 & Daejon & Chonju, Kimcheon, Chonan, Kongju \\
\cline { 2 - 5 } & Case 2 & Daejon & $\begin{array}{c}\text { Chonju, Kimcheon, Chonan, Kongju, } \\
\text { Daejon }\end{array}$ \\
\hline $\begin{array}{c}\text { Tracking } \\
\text { System }\end{array}$ & Case 3 & $\begin{array}{c}\text { Chonju, Kimcheon, } \\
\text { Chonan, Kongju }\end{array}$ & Daejon \\
\cline { 2 - 5 } & Case 4 & $\begin{array}{c}\text { Chonju, Kimcheon, } \\
\text { Chonan, Kongju, } \\
\text { Daejon }\end{array}$ & Daejon \\
\hline
\end{tabular}

\section{- Navigation System: The ground receiver is located in Daejon}

Case 1 and Case 2 of Table 4 fall into this category. For Case 1 where the ground receiver is located at Daejon while the airships are positioned at the altitude of $22 \mathrm{~km}$ over Chonju, Kimcheon, Chonan, and Kongju, HDOP results in 1.511. However, VDOP is 5.156. For Case 2 where one airship is added at Daejon to Case 1, HDOP has been improved to 1.268, while VDOP drops to 1.748. As our expectation, the added airship which is located at the right above the receiver position improves VDOP value much more than HDOP value. This means the vertical position accuracy would be much improved. All the DOP values for Case 1 and Case 2 are summarized in Table 5.

Table 5. DOP values for Case 1 and Case 2 as a Navigation System

\begin{tabular}{|l|l|l|l|l|l|}
\hline & GDOP & PDOP & HDOP & VDOP & TDOP \\
\hline \hline Case 1 & 5.763 & 5.373 & 1.511 & 5.156 & 2.103 \\
\hline Case 2 & 2.387 & 2.159 & 1.268 & 1.748 & 1.017 \\
\hline
\end{tabular}

\section{- Airship Tracking System: The stratospheric airships are located over Daejon}

This is the IGPS method. In this case the airship position is estimated by using the data collected by the receivers fixed on the ground for the purpose providing position information of airships to users through the navigation messages. With the stratospheric airship deployed at the altitude of $22 \mathrm{~km}$ over Daejon, Cases 3 and 4 are simulated as the same as in Table 4. In Case 3, with four ground-fixed receivers located at Chonju, Kimcheon, Chonan and Kongju, HDOP is 1.513 and VDOP is 5.242. When one more ground receiver is added at Daejon, HDOP is 1.272 and VDOP is 1.772 . Exactly the same reason as Case 1 and 
2 , because of the added receiver located at the right below the airship, VDOP value has been improved much. All the results suggest that the prediction of navigation airship positions by the ground observation can be fairly accurate. It is shown in Table 6 .

Table 6. DOP Values for Case 3 and Case 4 as an Airship Tracking System

\begin{tabular}{|c|c|c|c|c|c|}
\hline & GDOP & PDOP & HDOP & VDOP & TDOP \\
\hline \hline Case 3 & 5.874 & 5.456 & 1.513 & 5.242 & 2.170 \\
\hline Case 4 & 2.416 & 2.181 & 1.272 & 1.772 & 1.039 \\
\hline
\end{tabular}

\section{Simulation of Airship Using Navigation System Covering the Whole South KOREA Region}

This part of the paper deals with the deployment and the simulation of the airship navigation system covering the whole South Korea region. First, five areas are selected around major cities that are expected to attract demands for positioning information. Later, an extended navigation system is simulated on the basis of which the airship navigation system covers the whole South Korea region.

Those five areas are indicated in Figure 6 and each area is centered around one of the five major cities, i.e. Seoul, Daejon, Kwangju, Pusan, and Cheju.

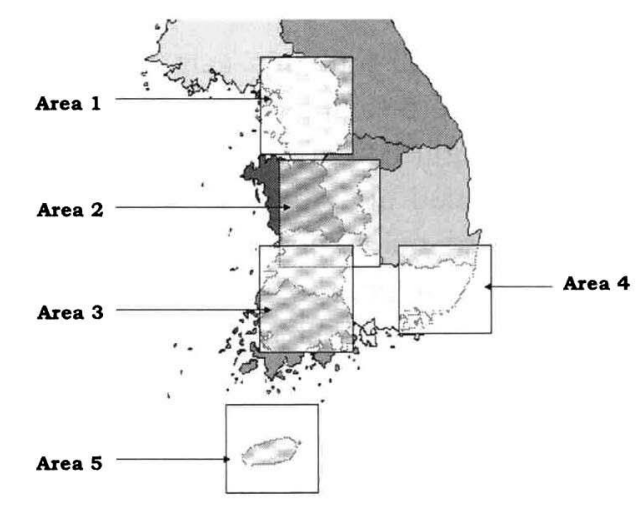

Fig. 6. Configuration of the Navigation System for Five Areas

The airships are deployed at each area at the same interval, i.e. 0.5 degrees longitudinally and 0.5 degrees latitudinally. Also extra airships are added to major city areas in precaution against the case when tall buildings block the transmitted signals and the required four or more airships cannot be secured.

\section{- The Navigation System simulation for Five Areas}

As shown in Table 7, the number of airships is determined at the five areas. And two central cities at each area are selected for simulation except Area 5. As each area is designed around each of five major cities, the deployment of the airships is determined in consideration of the observability at the populated areas. The results in Table 7 show satisfactory DOP values in all five areas. Table 8 shows the average DOP values of a user at Daejon for GPS with 15 degree mask angle, which can be used the reference DOP values. 
Table 7. DOP Values at Five Areas

\begin{tabular}{|c|c|c|c|c|c|c|}
\hline \multirow{2}{*}{ Area } & \multirow{2}{*}{$\begin{array}{c}\text { Number of } \\
\text { Airships }\end{array}$} & \multirow{2}{*}{$\begin{array}{c}\text { Receiver } \\
\text { position }\end{array}$} & \multicolumn{5}{|c|}{ DOP } \\
\cline { 5 - 7 } & \multirow{2}{*}{1} & GDOP & PDOP & HDOP & VDOP \\
\hline \hline \multirow{2}{*}{2} & \multirow{2}{*}{2} & Seoul & 2.226 & 1.987 & 0.908 & 1.767 \\
\cline { 3 - 7 } & \multirow{2}{*}{10} & Daejon & 2.147 & 1.969 & 0.761 & 1.816 \\
\cline { 3 - 7 } & & Chungju & 2.246 & 1.996 & 0.948 & 1.757 \\
\hline \multirow{2}{*}{3} & \multirow{2}{*}{10} & Kongju & 2.433 & 2.240 & 0.801 & 2.093 \\
\cline { 3 - 7 } & & Chonju & 2.850 & 2.469 & 1.353 & 2.065 \\
\hline \multirow{2}{*}{4} & \multirow{2}{*}{9} & Daegu & 8.337 & 6.772 & 3.864 & 5.561 \\
\cline { 3 - 7 } & & Pusan & 2.408 & 2.195 & 0.927 & 1.990 \\
\hline \multirow{2}{*}{5} & 6 & Cheju & 2.473 & 1.198 & 1.098 & 1.904 \\
\hline
\end{tabular}

Table 8. DOP Values of GPS at Daejon with 15 Degree Mask Angle

\begin{tabular}{|c|c|c|c|c|c|}
\hline & GDOP & PDOP & HDOP & VDOP & TDOP \\
\hline \hline Max & 5.750 & 4.645 & 2.312 & 4.382 & 3.390 \\
Min & 1.716 & 1.525 & 0.607 & 1.251 & 0.786 \\
Mean & 2.953 & 2.527 & 1.019 & 2.175 & 1.523 \\
\hline
\end{tabular}

\section{-Expansion to Cover the South KOREA}

For the next step the navigation system has been designed to cover the half of Korean peninsular on the basis of the above results regarding the five areas.

As shown in Figure 7, totally 64 airships are necessary to be deployed including six airships over Cheju Island alone. Following Figures 8 (a)-8(d) show the DOP contour lines over the South Korea drawn on the basis of the results of the airship deployment as Figure 7 The figures indicate that the DOP values at the boundaries greatly increase as the airship constellation slants toward one side.

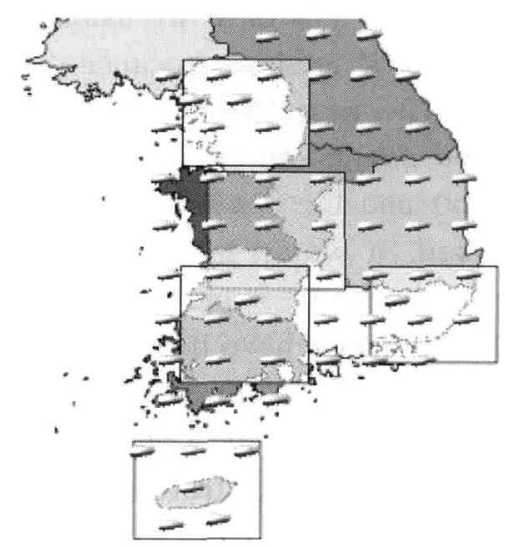

Fig. 7. Configuration of the Navigation System Covering South KOREA 


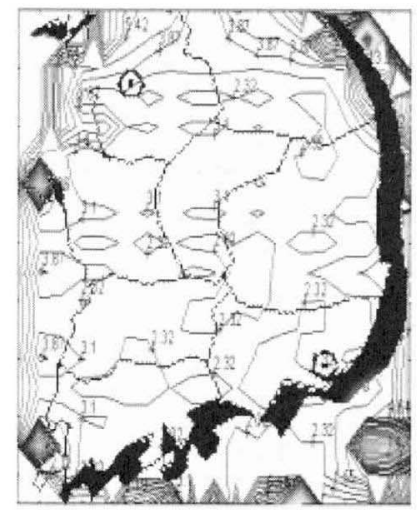

(a) GDOP Contour Lines

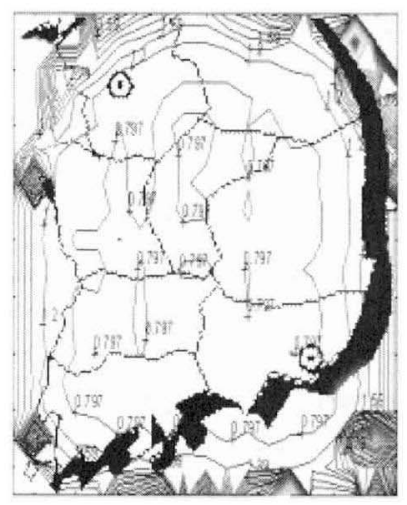

(c) HDOP Contour Line

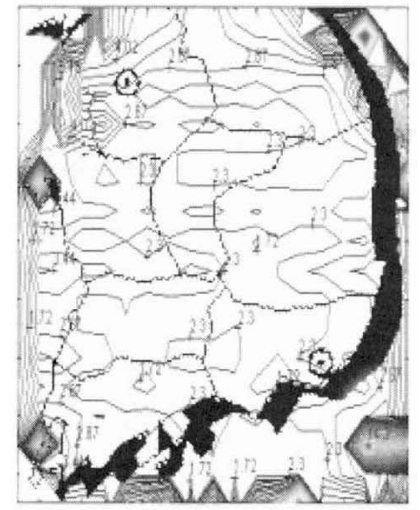

(b) PDOP Contour Lines

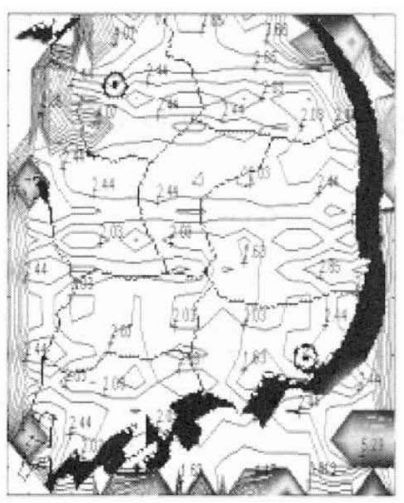

(d) VDOP Contour Line

Fig. 8. Contour Lines of DOP Values

\section{Signal Structure and Link Budget of Navigation System}

\section{Frequency Allocation}

Six frequency bands are assigned for the radionavigation satellites in Korea ${ }^{5}$. They are the bands of $149.9-150.05 \mathrm{MHz}, 399.9-400.05 \mathrm{MHz}, 1215-1260 \mathrm{MHz}, 1559-1610 \mathrm{MHz}$, $1610-1626.5 \mathrm{MHz}$, and $2483.5-2500 \mathrm{MHz}$. The first two bands seem not to be proper for the radionavigation system using stratospheric airships, because they are too narrow to be used for navigation signal transmission. The next two bands (i.e.1215-1260MHz and 1559$1610 \mathrm{MHz}$ ) seem preferable for the navigation purpose. so that these bands have been chosen for L1 (i.e. $1575.42 \pm 10 \mathrm{MHz}$ ) and L2 (i.e.1227.6 $\pm 10 \mathrm{MHz}$ ) of GPS. For GLONASS $1559-1610 \mathrm{MHz}$ band is being used. It would look appropriate to select these bands. However, there might exist some burden to GPS and GLONASS. The next band (i.e.1610$1626.5 \mathrm{MHz}$ ) is assigned for GLONASS, which have been fading out though. The last band of 2483.5-2500MHz seems also inappropriate since a great degree of interference is anticipated because of the wireless LAN's allocation $(2400-2483.5 \mathrm{MHz})$ is very close. As a conclusion, for the radionavigation system using stratospheric airships the frequency band can been determined at $1215-1260 \mathrm{MHz}$ or $1559-1626.5 \mathrm{MHz}$. As a conclusion, the frequency for the radionavigation system using the stratospheric airships $1595.44 \pm 10 \mathrm{MHz}$ is to be proposed. This frequency can be generated with 154 multiplication of the reference 
crystal frequency of $10.36 \mathrm{MHz}$. In this case, current P-code and C/A-code of GPS are $10.36 \mathrm{Mcps}$ and $1.036 \mathrm{Mcps}$, respectively.

\section{Transmitting Antenna}

Because the airships are at relatively low altitude comparing with that of LEO satellites the path length from transmitters to receivers is dramatically changed in the coverage of an airship as seen Figure 9. The ratio of the longest path to the shortest in the coverage could reach as large as 4 . It means that the power level fluctuation of the signal is as much as 16 times or $12 \mathrm{~dB}$. This kind of power level fluctuation can be minimized using a cosecantsquared beam pattern for the transmitting antenna as Figure $9^{6}$. For the cosecant-squared beam, the directive gain is gradually increasing along the direction from the beam center to the beam edge. If we set the directive gain to the beam edge $12 \mathrm{~dB}$ higher than to the beam center, the power level fluctuation could be cancelled out.

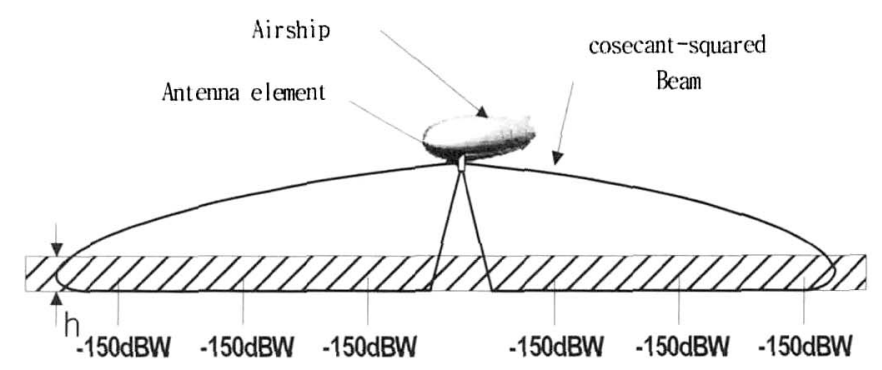

Fig. 9. Cosecant-squared beam pattern

\section{Link Budget}

For the link budget for the space segment of the stratospheric airships, the navigation system is to be designed differently from that of GPS. That is, a navigation system using stratospheric airship is totally domestic only in the sky of Korea and the frequency band of $1,595.44 \pm 10 \mathrm{MHz}$ has been selected. Especially, the band does not overlap L1 $(1,575.42 \pm 10 \mathrm{MHz})$ of GPS, the ground power level is not required to be maintained at the same level as GPS.

Considering the possible influence on the GPS receiver because the main frequency is only $20 \mathrm{MHz}$ apart from GPS signal, the ground power level has been set at $-150 \mathrm{dBW}$, which is about $10 \mathrm{~dB}$ higher than that of GPS. To obtain such a ground power level, the required transmitter power of the airship has been assumed to be $-20 \mathrm{dBW}$ as the amplifier output power. Since the stratospheric airships are at very low altitude, the amplifier output power does not need to be high. The feeder loss is set to $3 \mathrm{~dB}$, which is the same level as the GPS case. The transmitting antenna gain is determined for that the ground power level is maintained at a proper level and under the minimum level fluctuation in covered area. For this purpose, the antenna beam pattern should be designed to have low gain at the beam center and higher gain as it goes toward the beam edge.

As for the link budget for the path segment, as shown on lower part of Table 9 , the clear-sky free space path loss is to be divided into two parts for calculation, which are the beam center and the beam edge. The path length to the beam center is $22 \mathrm{~km}$. On the other hand, the beam edge is as long as $85 \mathrm{~km}$. This difference of the path lengths naturally causes the difference of the path losses. Adjusting the antenna beam pattern as mentioned earlier should compensate the loss difference. The precipitation loss margin due to rainfall and snowfall should be also considered, since airships are located in the stratosphere above the 
troposphere where the atmospheric phenomenon occurs. The antenna misalignment loss and the polarization mismatch loss are considered very low because it does not pass the ionosphere, which is one of major difference from the case of GPS. The details are summarized in Table 9.

Table 9. The Link Budget of the Stratospheric Airships

\begin{tabular}{|c|c|c|c|}
\hline Items & Unit & $\begin{array}{l}\text { Beam } \\
\text { Center }\end{array}$ & $\begin{array}{l}\text { Beam } \\
\text { Edge }\end{array}$ \\
\hline Frequency (L1) & $\mathrm{MHz}$ & $1,595.44$ & $1,595.44$ \\
\hline \multicolumn{4}{|c|}{ Space Segment (Stratospheric Airship) } \\
\hline Transmitter Power in Watts & W & 0.01 & 0.01 \\
\hline Transmitter Power in dBW & dBW & -20.0 & -20.0 \\
\hline Feeder Loss & $\mathrm{dB}$ & -3 & -3 \\
\hline Transmit Antenna Gain & $\mathrm{dBi}$ & 0.0 & 11.7 \\
\hline EIRP & dBW & -23.0 & -11.3 \\
\hline \multicolumn{4}{|l|}{ Path Segment } \\
\hline Path Length & $\mathrm{km}$ & 22 & 85 \\
\hline Clear-Sky Free Space Path Loss & $\mathrm{dB}$ & -123.4 & -135.1 \\
\hline Atmospheric Loss & $\mathrm{dB}$ & -0.1 & -.0 .1 \\
\hline Precipitation Loss Margin & $\mathrm{dB}$ & -2 & -2 \\
\hline Antenna Misalignment Loss & $\mathrm{dB}$ & -0.5 & -0.5 \\
\hline Polarization Mismatch Loss & $\mathrm{dB}$ & -1 & -1 \\
\hline Total Path Loss & $\mathrm{dB}$ & -127.0 & -138.7 \\
\hline Power Level on the Earth & $\mathrm{dBW}$ & -150 & -150 \\
\hline
\end{tabular}

\section{Conclusion}

GPS and GLONSS require not only high accuracy three dimensional position and velocity information but also clock information using relatively much less expensive receivers. However, some group of people might think that those two systems are currently serving civilians but their future availability could be uncertain, because they are originally military systems for the USA and Russia. For this and other reasons some countries have been endeavoring to own their own navigation systems. It has been about years since the importance of an alternative or a back-up navigation system other than GPS had been discussed in Korea. This paper deals with the airship deployment for the purpose of designing the navigation system appropriate for South Korea using a number of stratospheric airships. The basic simulation for the stratospheric airship navigation system has shown an excellent performance, compared with the case of GPS, and the basic simulation for the airship position determination using the IGPS method also has shown satisfactory DOP values. To design the navigation system using the stratospheric airships to cover the South Korea region on the basis of this simulation, first, five major areas have been determined for a basic simulation of airship deployment. And based on the result of the simulation, the airship constellation with 64 airships to cover the whole South Korea region is simulated and analyzed. From the above simulation it has been possible to obtain satisfactory DOP values from the South Korea region. For the last part this paper, the frequency allocation, the link budget and antenna beam pattern for the stratospheric airship at the altitude of $22 \mathrm{~km}$ from ground have been calculated and analyzed. 
This paper has suggested the possibility of the navigation system using stratospheric airships in South Korea region, and reviewed its feasibility through simulation of the airship deployment and the visibility range with respect to the mask angles in case the stratospheric airships are located at the altitude of $22 \mathrm{~km}$.

\section{Reference}

1. B. Hofmann-Wellenhof, Global Positioning System Theory and Practice, Springer Wien New York, 1997, pp. 273-277.

2. Bradford W. Parkinson, Global Positioning System: Theory and Applications, Vol. I, Progress in Astronautics and Aeronautics, Washington DC, 1996, Chapter 5.

3. Bradford W. Parkinson, Global Positioning System: Theory and Applications, Vol. I, Progress in Astronautics and Aeronautics, Washington DC, 1996, Chapter 11.

4. Bradford W. Parkinson, Global Positioning System: Theory and Applications, Vol. II, Progress in Astronautics and Aeronautics, Washington DC, 1996, p. 584.

5. Korean Ministry of Information and Telecommunication, Table of Radio Frequency Allocations, Seoul, Korea, 1996.

6. Merill I. Skolnik, Introduction to Radar Systems, $2^{\text {nd }}$ Edition, Singapore, McGraw Hill 1988. 